\title{
Transmuted Lomax Distribution
}

\author{
S.K. Ashour ${ }^{1, *}$, M.A. Eltehiwy ${ }^{2}$ \\ Institute of Statistical Studies \& Research, Cairo University, Egypt \\ *Corresponding author: ashoursamir@hotmail.com
}

Received July 04, 2013; Revised December 15, 2013; Accepted December 20, 2013

\begin{abstract}
A generalization of the Lomax distribution so-called the transmuted Lomax distribution is proposed and studied. Various structural properties including explicit expressions for the moments, quantiles, and mean deviations of the new distribution are derived. The estimation of the model parameters is performed by maximum likelihood method. We hope that the new distribution proposed here will serve as an alternative model to the other models which are available in the literature for modeling positive real data in many areas.
\end{abstract}

Keywords: Lomax distribution, hazard rate function, reliability function, parameter estimation

Cite This Article: S.K. Ashour, and M.A. Eltehiwy, “Transmuted Lomax Distribution.” American Journal of Applied Mathematics and Statistics 1, no. 6 (2013): 121-127. doi: 10.12691/ajams-1-6-3.

\section{Introduction}

The quality of the procedures used in a statistical analysis depends heavily on the assumed probability model or distributions. Because of this, considerable effort has been expended in the development of large classes of standard probability distributions along with revelent statistical methodologies. In fact, the statistics literature is filled with hundreds of continuous univariate distributions. However, in recent years, applications from the environmental, financial, biomedical sciences, engineering among others, have further shown that data sets following the classical distributions are more often the exception rather than the reality. Since there is a clear need for extended forms of these distributions a significant progress has been made toward the generalization of some well-known distributions and their successful application to problems in areas such as engineering, finance, economics and biomedical sciences, among others.

In this article we use transmutation map approach suggested by Shaw and Buckley [8] to define a new model which generalizes the Lomax model. We will call the generalized distribution as the transmuted Lomax (TL) distribution. According to the Quadratic Rank Transmutation Map, (QRTM), approach the cumulative distribution function (cdf) satisfy the relationship

$$
F_{2}(x)=(1+\lambda) F_{1}(x)-\lambda F_{1}(x)^{2}
$$

which on differentiation yields,

$$
f_{2}(x)=f_{1}(x)\left[1+\lambda-2 \lambda F_{1}(x)\right]
$$

where $f_{1}(x)$ and $f_{2}(x)$ are the corresponding probability density function (pdf) associated with $F_{1}(x)$ and $F_{2}(x)$ respectively and $-1 \leq \lambda \leq 1$. An extensive information about the quadratic rank transmutation map is given in [8].
We will use the above formulation for a pair of distributions $F(x)$ and $G(x)$ where $G(x)$ is a submodel of $F(x)$ therefore, a random variable $X$ is said to have a transmuted probability distribution with cdf $F(x)$ if

$$
F(x)=(1+\lambda) G(x)-\lambda G(x)^{2},|\lambda| \leq 1
$$

where $G(x)$ is the cdf of the base distribution. Observe that at $\lambda=0$ we have the distribution of the base random variable. Aryal and Tsokos [1,2] studied the transmuted extreme distributions. The authors provided the mathematical characterization of transmuted Gumbel and transmuted Weibull distributions and their applications to analyze real data sets. Aryal [3] studied the transmuted log- logistic distribution and discussed some properties of this family. In the present study we will provide mathematical formulation of the transmuted Lomax (TL) distribution and some of its properties.

\section{Transmuted Lomax Distribution}

The Lomax distribution, conditionally also called the Pareto Type II distribution, is a heavy-tail probability distribution often used in business, economics, and actuarial modeling. It was used by Lomax [6] to fit data in business failure. It is essentially a Pareto distribution that has been shifted so that its support begins at zero.

A random variable $\mathrm{X}$ is said to have a Lomax distribution with parameters $\theta$ and $\gamma>0$ if its probabability density function (pdf) is given by

$$
\mathrm{g}(\mathrm{x})=\alpha \theta \gamma(1+\gamma \mathrm{x})^{-(\theta+1)} x>0, \theta \text { and } \gamma>0
$$

and the cdf of $\mathrm{X}$ is given by

$$
G(x)=\left[1-(1+\gamma x)^{-\theta}\right], x>0, \theta \text { and } \gamma>0
$$


Now using (3) and (5) we have the cdf of a transmuted Lomax

$$
\left.F(x)=\left[1-(1+\gamma x)^{-\theta}\right] \times\left(\begin{array}{l}
(1+\lambda) \\
-\lambda\left[1-(1+\gamma x)^{-\theta}\right.
\end{array}\right]\right)
$$

Hence, the pdf of the transmuted Lomax distribution with parameters $\theta, \gamma$ and $\lambda$ is

$$
f(x)=\frac{\alpha \theta \gamma}{(1+\gamma x)^{(\theta+1)}}\left(1+\lambda-2 \lambda\left[1-(1+\gamma x)^{-\theta}\right]\right) .
$$

Note for $\lambda=0$ and $\gamma=1$, we have the pdf of Lomax distribution and transmuted Pareto distribution respectively. Figure 1 illustrates some of the possible shapes of the density function of transmuted Lomax distribution for selected values of the parameters.
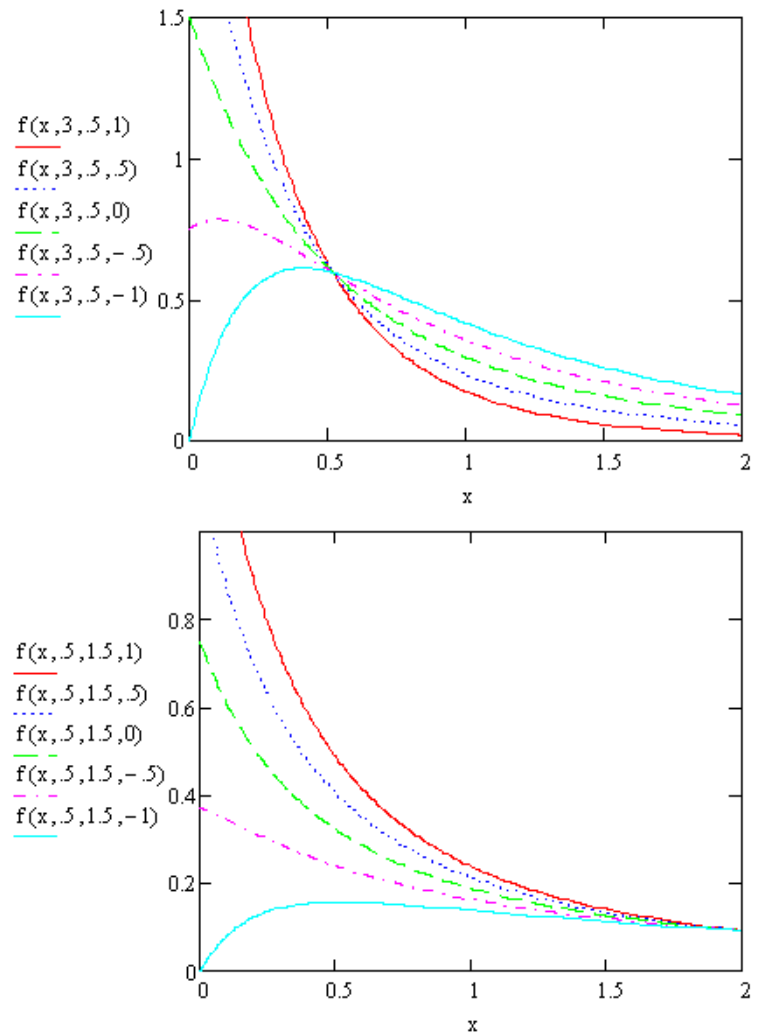

Figure 1. the pdf of TL distrbution for $\theta=3,0.5, \gamma=0.5,1.5$ when $\lambda=-1,-0.5,0,0.5,1$

\section{Moments and Quantiles}

In this section we shall present the moments and quantiles for the transmuted Lomax distribution. The $r^{\text {th }}$ moments of a transmuted Lomax random variable $X$, is given by

$$
\begin{aligned}
& E\left(X^{r}\right)=\int_{0}^{\infty} \frac{\alpha \theta \gamma \mathrm{x}^{r}}{(1+\gamma \mathrm{x})^{(\theta+1)}}\left(\begin{array}{c}
1+\lambda \\
-2 \lambda\left[1-(1+\gamma x)^{-\theta}\right]
\end{array}\right) \\
& =\frac{(1+\lambda)}{\gamma^{r}} \sum_{i=0}^{r}\left(\begin{array}{l}
r \\
i
\end{array}\right)(-1)^{i} \frac{\theta}{\theta+i-r} \\
& -\frac{2 \lambda}{\gamma^{r}} \sum_{i=0}^{r}\left(\begin{array}{l}
r \\
i
\end{array}\right)(-1)^{i} \frac{\theta^{2}}{(2 \theta+i-r)(\theta+i-r)}
\end{aligned}
$$

The first three moments can be obtain by taking $r=1,2$ and 3 in (8) as follows:

$$
\begin{gathered}
\mu_{1}^{\prime}=\frac{\theta(2-\lambda)-1}{2 \gamma \theta^{2}-3 \gamma \theta+\gamma}, \\
\mu_{2}^{\prime}=\frac{\theta(4-3 \lambda)-2}{\gamma^{2}\left(2 \theta^{3}-7 \theta^{2}+7 \theta-2\right)},
\end{gathered}
$$

and

$$
\begin{aligned}
\mu_{3}^{\prime}= & \frac{6(\lambda+1)}{\gamma^{3}\left(\theta^{3}-6 \theta^{2}+11 \theta-6\right)} \\
& -\frac{9 \lambda\left(5 \theta^{2}-9 \theta+2\right)}{\gamma^{3}\left(4 \theta^{5}-32 \theta^{4}+95 \theta^{3}-130 \theta^{2}+81 \theta-18\right)}
\end{aligned}
$$

The variance and coefficient of variation (CV) for TL are

$$
\begin{gathered}
\operatorname{Var}(X)=\frac{\theta^{3}\left(4-2 \lambda-\lambda^{2}\right)+\theta^{2}\left(2 \lambda^{2}-\lambda-4\right)+\theta(\lambda+1)}{\gamma^{2}(\theta-2)\left(2 \theta^{2}-3 \theta+1\right)^{2}}, \\
\text { for } \theta>2
\end{gathered}
$$

and

$$
\begin{gathered}
C V=\frac{\sqrt{\theta^{3}\left(4-2 \lambda-\lambda^{2}\right)+\theta^{2}\left(2 \lambda^{2}-\lambda-4\right)+\theta(\lambda+1)}}{\sqrt{(\theta-2)}(2 \theta-\theta \lambda-1)} . \\
\text { for } \theta>2
\end{gathered} .
$$

The skewness and kurtosis measures can be obtained from expressions

$$
\begin{gathered}
\text { Skewness }=\frac{\mu_{3}^{\prime}-3 \mu_{2}^{\prime} \mu+2 \mu^{3}}{\sigma^{3}} \\
\text { Kurtosis }=\frac{\mu_{4}^{\prime}-3 \mu_{3}^{\prime} \mu+6 \mu_{2}^{\prime} \mu^{2}-3 \mu^{4}}{\sigma^{4}}
\end{gathered}
$$

Upon substituting in the raw moments. Figure 2 shows the skewness and kurtosis of the TL distribution as a function of $\lambda$ for $\theta=10, \gamma=1$.
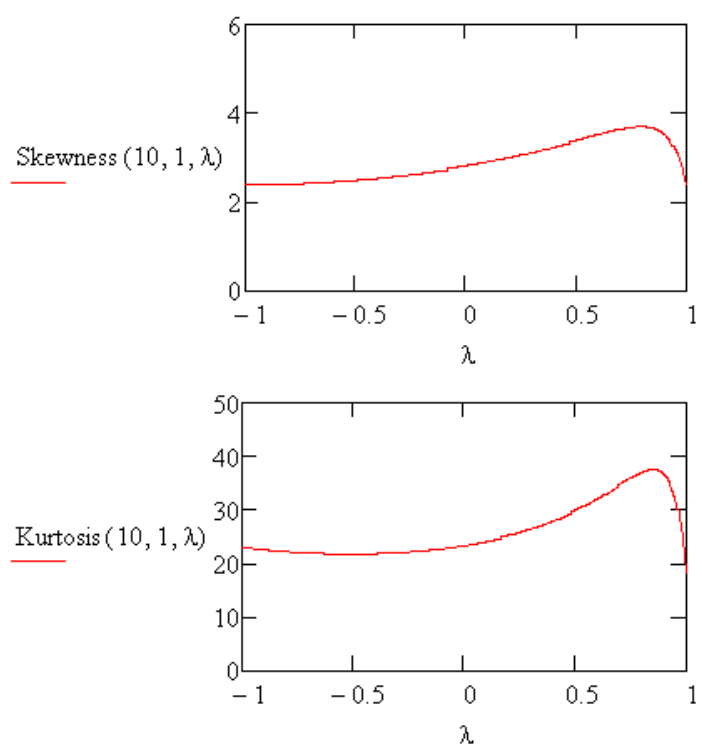

Figure 2. skewness and kurtosis of TL distribution as a function of $\lambda$ and for $\theta=10, \gamma=1$. 
We can obtain the estimator of $\theta, \gamma$ and $\lambda$ by using the following equations:

$$
\mu_{r}^{\prime}=\frac{\sum_{i=0}^{n} x_{i}^{r}}{n}, r=1,2,3
$$

i.e.,

$$
\begin{gathered}
\frac{\tilde{\theta}(2-\tilde{\lambda})-1}{2 \tilde{\gamma} \tilde{\theta}^{2}-3 \tilde{\gamma} \tilde{\theta}+\tilde{\gamma}}=\bar{x} \\
\frac{\tilde{\theta}(4-3 \tilde{\lambda})-2}{\tilde{\lambda}^{2}\left(2 \tilde{\theta}^{3}-7 \tilde{\theta}^{2}+7 \tilde{\theta}-2\right)}=\frac{\sum_{i=0}^{n} x_{i}^{2}}{n} \\
\frac{6(\tilde{\lambda}+1)}{\tilde{\lambda}^{3}\left(\tilde{\theta}^{3}-6 \tilde{\theta}^{2}+11 \tilde{\theta}-6\right)} \\
-\frac{9 \tilde{\lambda}\left(5 \tilde{\theta}^{2}-9 \tilde{\theta}+2\right)}{\tilde{\lambda}^{3}\left(4 \tilde{\theta}^{5}-32 \tilde{\theta}^{4}+95 \tilde{\theta}^{3}-130 \tilde{\theta}^{2}+81 \tilde{\theta}-18\right)} \\
=\frac{\sum_{i=0}^{n} x_{i}^{3}}{n}
\end{gathered}
$$

We can obtain $\tilde{\theta}, \tilde{\gamma}$ and $\tilde{\lambda}$ by solving nonlinear equation (14), (15) and (16) numerically.

The $q^{\text {th }}$ quantile $x_{q}$ of the transmuted Lomax distribution can be obtaned from (6) as

$$
\frac{\left[\frac{\lambda-1+\sqrt{(1+\lambda)^{2}-4 \lambda q}}{2 \lambda}\right]^{-1 / \theta}-1}{\gamma}
$$

Hence, the distribution median is

$$
x_{0.5}=\frac{\left[\frac{\lambda-1+\sqrt{1+\lambda^{2}}}{2 \lambda}\right]^{-1 / \theta}-1}{\gamma}
$$

To illustrate the effect of the shape parameter $\lambda$ on skewness and kurtosis we consider measures based on quantiles. The shortcomings of the classical kurtosis and skewness measures are well known. There are many heavy-tailed distributions for which this measure is infinite, so it becomes uninformative. The Bowley's skewness [5] is one of the earliest skewness measures defined by the average of the quartiles minus the median, divided by the half the interquartile range, given by

$$
\mathcal{B}=\frac{Q_{3}+Q_{1}-2 Q_{2}}{Q_{3}-Q_{1}}=\frac{Q(3 / 4)+Q(1 / 4)-2 Q(2 / 4)}{Q(3 / 4)-Q(1 / 4)},
$$

and the Moors kurtosis [7] is based on octiles and is given by

$$
\begin{aligned}
\mathcal{M} & =\frac{\left(E_{3}-E_{1}\right)+\left(E_{7}-E_{5}\right)}{E_{6}-E_{2}} \\
& =\frac{Q(3 / 8)-Q(1 / 8)+Q(7 / 8)-Q(5 / 8)}{Q(6 / 8)-Q(2 / 8)},
\end{aligned}
$$

For any distribution symmetrical to 0 the Moors kurtosis reduces to

$$
\mathcal{M}=\frac{\left(E_{7}-E_{5}\right)}{E_{6}} .
$$

It is easy to calculate that for standand normal distribution $E_{1}=-E_{7}=-1.15, E_{2}=-E_{6}=-0.32$. Therefore, $\mathcal{M}=1.23$. Hence, the centered Moor's coefficient is given by

$$
\mathcal{M}=\frac{\left(E_{3}-E_{1}\right)+\left(E_{7}-E_{5}\right)}{E_{6}-E_{2}}-1.23 .
$$

Fiqure 3 displays the Bowley $(\mathcal{B})$ and Moors $(\mathcal{M})$ kurtosis as a function of the parmeter $0<\lambda \leq 1$ and $-1 \leq \lambda<0$ for $\gamma=1$ and $\theta=10$. It is evident that both measures depend on the parameter $\lambda$.
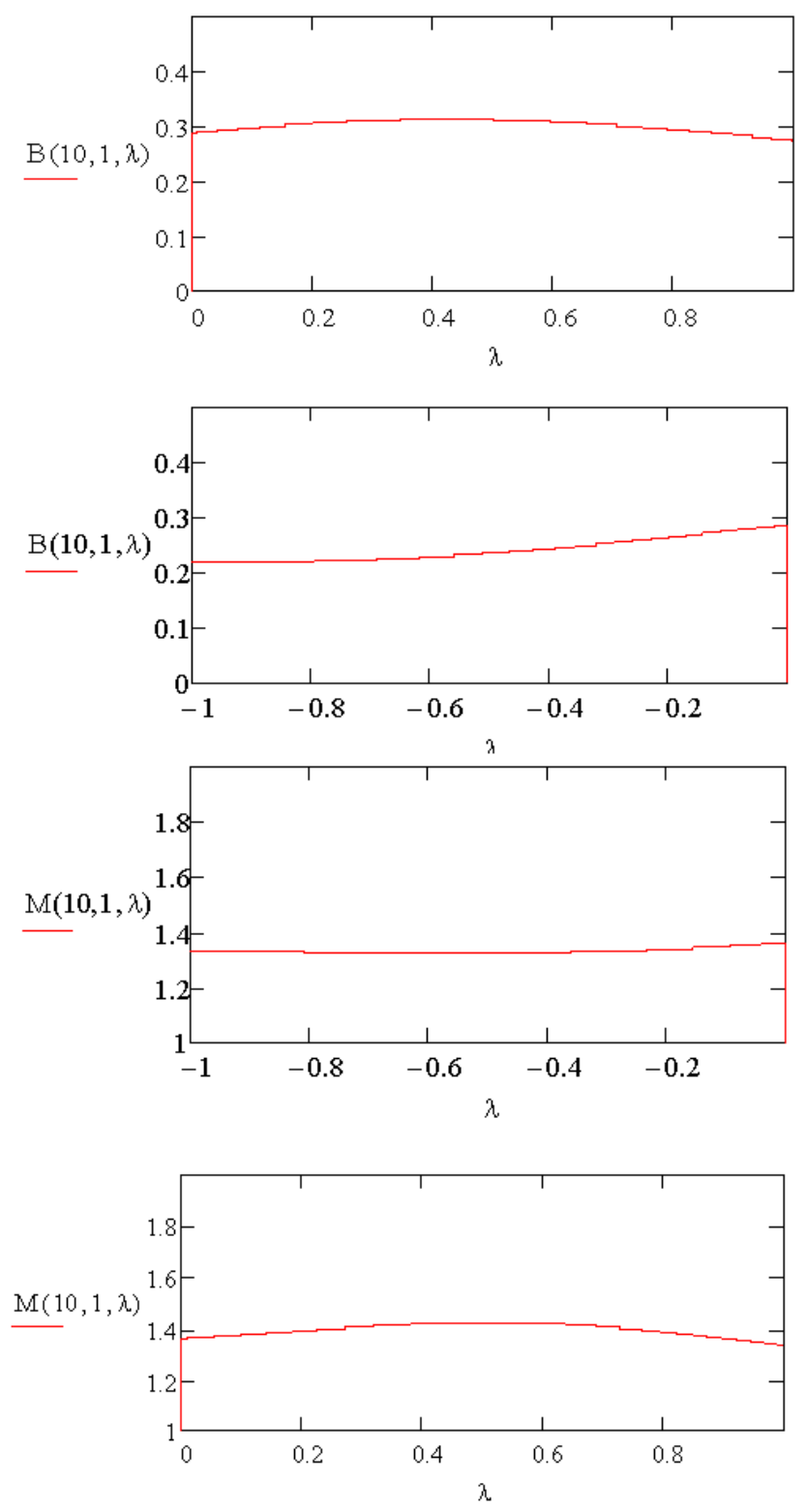

Figure 3. Behavior of Bowley(B) and Moors(M) kurtosis for TE

\section{Mean Deviation}

The amount of scatter in a population is evidently measured to some extent by the totality of deviations from the mean and the median. These are known as the mean 
deviation about the mean and the mean deviation about the median respectively and are defined by

$$
\delta_{1}=\int_{0}^{\infty}|x-\mu| f(x) d x
$$

and

$$
\delta_{2}=\int_{0}^{\infty}|x-M| f(x) d x
$$

where

$$
\mu=E(X)=\frac{\theta(2-\lambda)-1}{2 \gamma \theta^{2}-3 \gamma \theta+\gamma}
$$

and

$$
M=\operatorname{Median}(X)=\frac{\left[\frac{\lambda-1+\sqrt{1+\lambda^{2}}}{2 \lambda}\right]^{-1 / \theta}-1}{\gamma} .
$$

The measures $\delta_{1}(X)$ and $\delta_{2}(X)$ can be expressed as $\delta_{1}(X)=2 \mu F(\mu)-2 J(\mu) \quad$ and $\quad \delta_{2}(X)=\mu-2 J(M)$ where $J(q)=\int_{0}^{q} x f(x) d x$. For a transmuted Lomax

$$
\begin{aligned}
J(q) & =(1+\lambda) \theta \gamma \int_{0}^{q} x \frac{\alpha \theta \gamma}{(1+\gamma \mathrm{x})^{(\theta+1)}} d x \\
& -2 \lambda \theta \gamma \int_{0}^{q} x \frac{\theta \gamma\left[1-(1+\gamma \mathrm{x})^{-\theta}\right]}{(1+\gamma \mathrm{x})^{(\theta+1)}} d x
\end{aligned}
$$

One can easily compute these integrals numerically in software such as Mathcad [4] and hence get the mean deviations about the mean and about the median as desired. From the mean deviations we can construct Lorenz and Bonferroni curves, which are used in several areas including economics, reliability, insurance and medicine and others.

Some numerical values of the mean deviation from mean and median for selected value of for $\gamma=1$ and $\theta=10$ and different values of $\lambda$ are listed in the Table 1 below.

Table 1. Mean deviation from the mean and the median for selected values of the parameters

\begin{tabular}{|l|c|c|c|c|c|}
\hline & $\lambda=-1$ & $\lambda=-0.5$ & $\lambda=0$ & $\lambda=0.5$ & $\lambda=1$ \\
\hline$\delta_{1}$ & 0.103 & 0.098 & 0.086 & 0.067 & 0.040 \\
\hline$\delta_{2}$ & 0.098 & 0.092 & 0.111 & 0.061 & 0.037 \\
\hline
\end{tabular}

\section{Random Number Generation and Parameter Estimation}

Using the method of inversion we can generate random numbers from the transmuted Lomax distribution as

$$
\left[1-(1+\gamma x)^{-\theta}\right]\left((1+\lambda)-\lambda\left[1-(1+\gamma x)^{-\theta}\right]\right)=u
$$

where $u \sim U(0,1)$. After simple calculation this yields

$$
x=\frac{\left[\frac{\lambda-1+\sqrt{(1+\lambda)^{2}-4 \lambda u}}{2 \lambda}\right]^{-1 / \theta}-1}{\gamma}
$$

One can use equation (22) to generate random numbers when the parameters $\theta, \gamma$ and $\lambda$ are known. The maximum likelihood estimates, MLEs, of the parameters that are inherent within in the transmuted Lomax probability distribution function is given by the following:

Let $X_{1}, X_{2}, \ldots, X_{n}$ be a sample size $\mathrm{n}$ from a transmuted Lomax distribution. Then the likelihood function is given by

$$
L=\frac{\theta^{n} \gamma^{n} \prod_{i=1}^{n}\left(1+\lambda-2 \lambda\left[1-(1+\gamma x)^{-\theta}\right]\right)}{\prod_{i=1}^{n}(1+\gamma x)^{(\theta+1)}}
$$

Hence, the log-likelihood function $\mathcal{L}=\ln L$ becomes

$$
\begin{aligned}
\mathcal{L}= & n(\ln \theta+\ln \gamma)-n(\theta+1) \ln (1+\gamma \mathrm{x}) \\
& +\sum_{i=1}^{n} \ln \left(1+\lambda-2 \lambda\left[1-(1+\gamma x)^{-\theta}\right]\right)
\end{aligned}
$$

Therefore, the MLEs of $\theta, \gamma$ and $\lambda$ which maximize (23) must satisfy the following normal equations:

$$
\begin{gathered}
\frac{\partial \mathcal{L}}{\partial \theta}=\frac{n}{\theta}-\operatorname{nln}(1+\gamma \mathrm{x})+\sum_{i=1}^{n} \frac{2 \lambda(1+\gamma \mathrm{x})^{-\theta} \ln (1+\gamma \mathrm{x})}{\left(1+\lambda-2 \lambda\left[1-(1+\gamma x)^{-\theta}\right]\right)}, \\
\frac{\partial \mathcal{L}}{\partial \gamma}=\frac{n}{\gamma}-\frac{n(\theta+1) x}{(1+\gamma \mathrm{x})}+\sum_{i=1}^{n} \frac{2 \lambda \gamma \theta(1+\gamma \mathrm{x})^{-(\theta+1)}}{\left(1+\lambda-2 \lambda\left[1-(1+\gamma x)^{-\theta}\right]\right)}, \\
\frac{\partial \mathcal{L}}{\partial \lambda}=\sum_{i=1}^{n} \frac{1-2\left[1-(1+\gamma x)^{-\theta}\right]}{\left(1+\lambda-2 \lambda\left[1-(1+\gamma x)^{-\theta}\right]\right)}
\end{gathered}
$$

The MLE of $\theta, \gamma$ and $\lambda$ is obtained by solving this nonlinear system of equations. Setting these expressions to zero and solving them simultaneously yields the maximum likelihood estimates of the three parameters.

\section{Reliability Analysis}

The survival function, also known as the reliability function in engineering, is the characteristic of an explanatory variable that maps a set of events, usually associated with mortality or failure of some system onto time. It is the probability that the system will survive beyond a specified time.

The transmuted Lomax distribution can be a useful model to characterize failure time of a given system because of the analytical structure. The reliability function $R(t)$, which is the probability of an item not failing prior to sometime $t$, is defined by $R(t)=1-F(t)$, The reliability function of a transmuted Lomax distribution is given by 


$$
\left.R(t)=1-\left[1-(1+\gamma t)^{-\theta}\right] \times\left(\begin{array}{l}
(1+\lambda) \\
-\lambda\left[1-(1+\gamma t)^{-\theta}\right.
\end{array}\right]\right)
$$

The other characteristic of interest of a random variable is the hazard rate function also known as instantaneous failure rate defined by

$$
h(t)=\frac{f(t)}{1-F(t)}
$$

which is an important quantity characterizing life phenomenon. It can be loosely interpreted as the conditional probability of failure, given it has survived to the time $t$.

The hazard rate function for a transmuted Lomax distribution is given by

$$
h(t)=\frac{\theta \gamma\left(1+\lambda-2 \lambda\left[1-(1+\gamma t)^{-\theta}\right]\right)(1+\gamma t)^{-(\theta+1)}}{\left[1-\left[1-(1+\gamma t)^{-\theta}\right]\left((1+\lambda)-\lambda\left[1-(1+\gamma t)^{-\theta}\right]\right)\right]}
$$
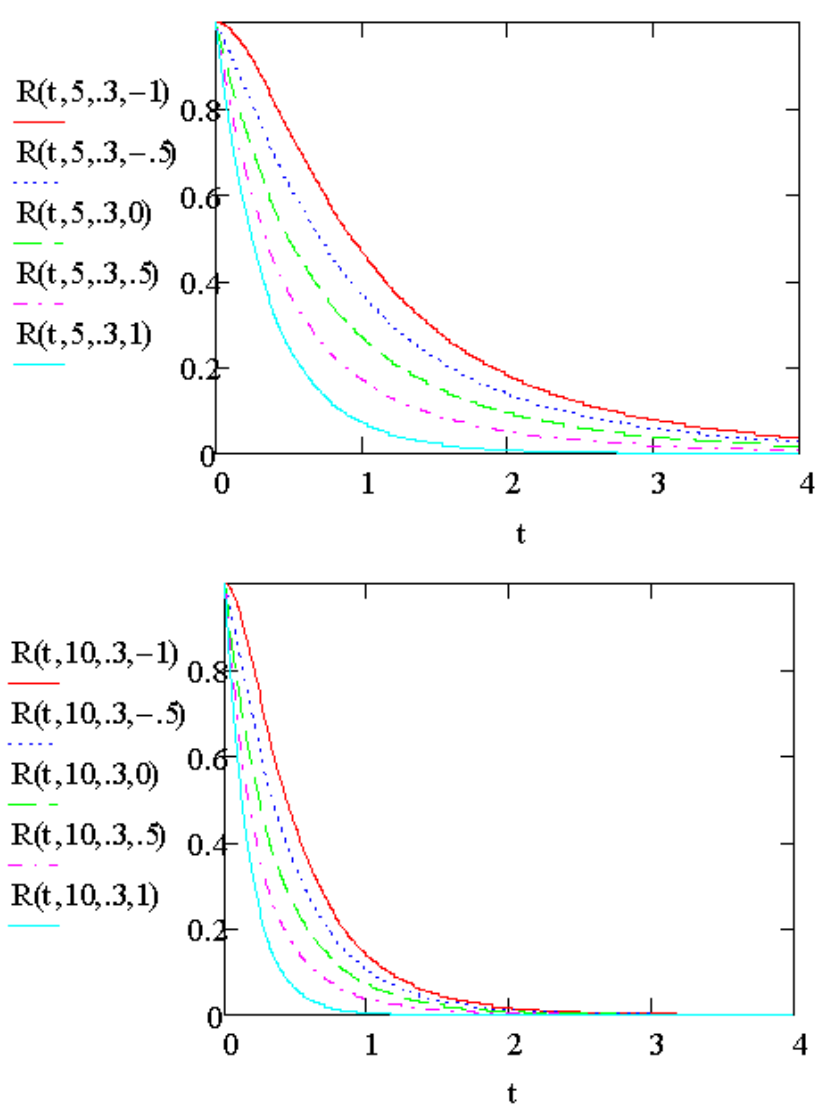

Figure 4. The reliability function of TL distrbution for $\theta=5,10, \gamma=0.3$ when $\lambda=-1,-0.5,0,0.5,1$

It is important to note that the units for $h(x)$ is the probability of failure per unit of time, distance or cycles. Figure 5 illustrates the behavior of the hazard rate function of a transmuted Lomax distribution for selected values of the parameters.

Observing the behavior of the hazard rate function it is worth noting that the transmuted Lomax distribution will have more applicability than the Lomax distribution and some of its generalizations.

The hazard rate function of the transmuted Lomax distribution has the following properties: i. If $\gamma=1$ the failure rate is same as the transmuted Pareto II distribution

$$
h(t)=\frac{\theta \gamma\left(1+\lambda-2 \lambda\left[1-(1+t)^{-\theta}\right]\right)(1+\mathrm{t})^{-(\theta+1)}}{\left[(1+t)^{-\theta}\left(1+\lambda-\lambda\left[1-(1+t)^{-\theta}\right]\right)\right]}
$$

ii. If $\lambda=0$ the failure rate is same as the Lomax distribution

$$
h(t)=\theta \gamma(1+\gamma \mathrm{t})^{-1}
$$

iii. If $\lambda=0, \gamma=1$ he failure rate is same as the Pareto II distribution.

$$
h(t)=\theta(1+\mathrm{t})^{-1}
$$
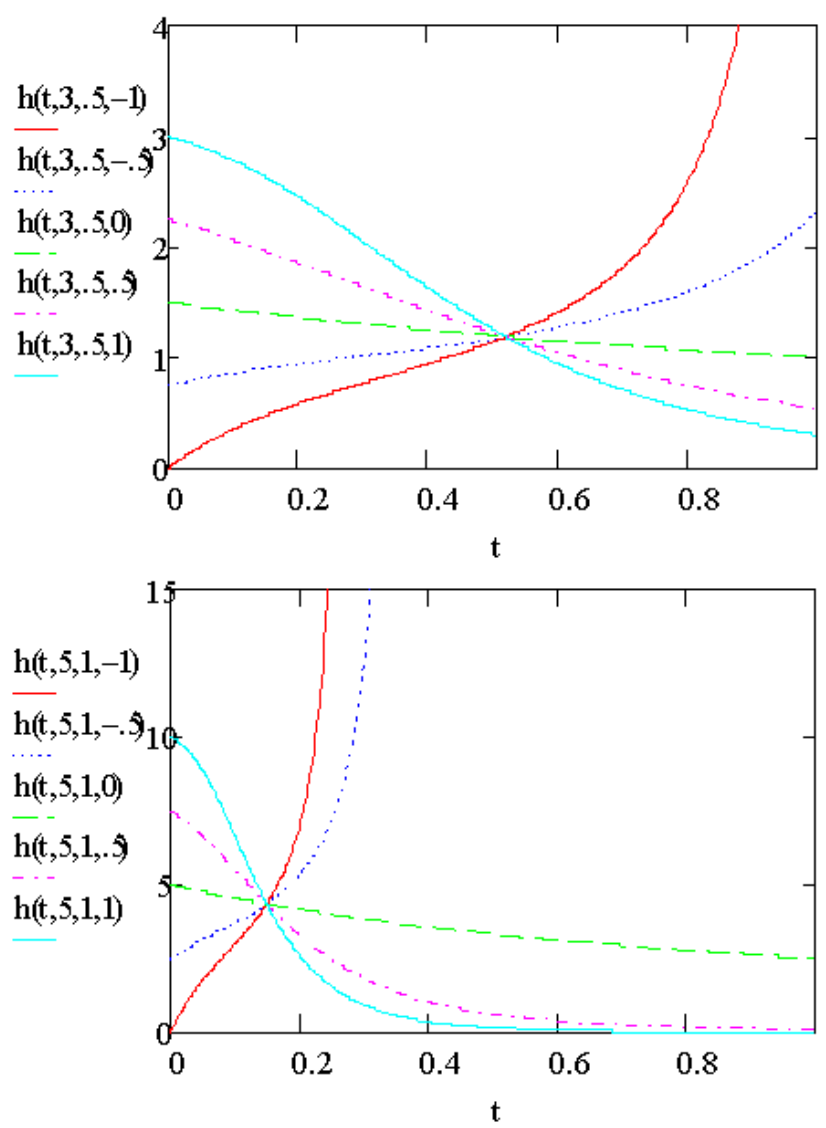

Figure 5. The Hazard rate function of TL for $\theta=3,5, \gamma=0.5,1$ when $\lambda=-1,-0.5,0,0.5,1$

Many generalized probability models have been proposed in reliability literature through the fundamental relationship between the reliability function $R(x)$ and its cumulative hazard function (CHF) $H(t)$ given by $-\ln R(x)$. The CHF describes how the risk of a particular outcome changes with time. The cumulative hazard rate function of a transmuted Lomax distribution is given by

$$
H(t)=-\ln \left(1-\left[1-(1+\gamma t)^{-\theta}\right]\left(\begin{array}{l}
(1+\lambda) \\
-\lambda\left[1-(1+\gamma t)^{-\theta}\right]
\end{array}\right)\right)
$$

\section{Observe that:}

$$
\text { i. } H(t) \text { is nondecreasing for all } t \geq 0 \text {, }
$$



ii. $H(0)=0$,
iii. $\lim _{t \rightarrow \infty} H(t)=\infty$

It is important to note that the units for $H(t)$ are the cumulative probability of failure per unit of time, distance or cycles.

\section{Order Statistics}

Order statistics make their appearance in many areas of statistical theory and practice. We know that if $X_{(1)}, X_{(2)}, \ldots, X_{(n)}$ denotes the order statistics of a random sample $X_{1}, X_{2}, \ldots, X_{n}$, from a continuous population with cdf $F_{X}(x)$ and pdf $f_{X}(x)$, then the pdf of $X_{(j)}$ is given by

$$
\begin{aligned}
& f_{X_{(j)}}(x) \\
= & \frac{n !}{(j-1) !(n-j) !} f_{X}(x)\left[F_{X}(x)\right]^{j-1} \times\left[1-F_{X}(x)\right]^{n-j}
\end{aligned}
$$

For $j=1,2, \ldots, n$.

We have from (4) and (5) the pdf of the $j^{\text {th }}$ order Lomax random variable $X_{(j)}$ is given by

$$
\begin{aligned}
& \mathrm{g}_{X_{(j)}}(x) \\
& =\frac{n ! \theta \gamma(1+\gamma \mathrm{x})^{-(\theta+1)}}{(j-1) !(n-j) !}\left[1-(1+\gamma \mathrm{x})^{-\theta}\right]^{(\mathrm{j}-1)} \\
& \quad \times\left\{1-\left[1-(1+\gamma \mathrm{x})^{-\theta}\right]\right\}^{n-j}
\end{aligned}
$$

Therefore, the pdf of the $n^{\text {th }}$ order Lomax statistic $X_{(n)}$ is given by

$$
\mathrm{g}_{X_{(n)}}(x)=n \theta \gamma(1+\gamma \mathrm{x})^{-(\theta+1)}\left[1-(1+\gamma \mathrm{x})^{-\theta}\right]^{(\mathrm{n}-1)}
$$

and the pdf of the first order Lomax statistic $X_{(1)}$ is given

$$
\mathrm{g}_{X_{(1)}}(x)=n \theta \gamma(1+\gamma \mathrm{x})^{-(\theta+1)}\left\{(1+\gamma \mathrm{x})^{-\theta}\right\}^{n-1}
$$

Note that in a particular case of $n=2$, (25) yields

$$
\mathrm{g}_{X_{(2)}}(x)=2 \theta \gamma(1+\gamma \mathrm{x})^{-(\theta+1)}\left[1-(1+\gamma \mathrm{x})^{-\theta}\right]
$$

and (26) yields

$$
\mathrm{g}_{X_{(1)}}(x)=2 \theta \gamma(1+\gamma \mathrm{x})^{-(2 \theta+1)}
$$

Observe that (27) and (28) are special cases of (7) for $\lambda=-1$ and $\lambda=1$ respectively. It has been observe that a transmuted Lomax distribution with $\lambda=1$ is the distribution of $\min \left(X_{1}, X_{2}\right)$ and a transmuted Lomax with $\lambda=-1$ is the of the $\max \left(X_{1}, X_{2}\right)$ where $X_{1}$ and
$X_{2}$ are independent and identically distributed Lomax random variables.

Now we provide the distribution of the order statistics for transmuted Lomax random variable. The pdf of the $j^{\text {th }}$ order statistic for transmuted Lomax distribution is given by

$$
\begin{aligned}
& f_{X_{(j)}}(x) \\
= & \frac{n !}{(j-1) !(n-j) !} \frac{\theta \gamma\left[1-(1+\gamma x)^{-\theta}\right]^{j-1}}{(1+\gamma x)^{(\theta+1)}} \\
& \times\left(1+\lambda-2 \lambda\left[1-(1+\gamma x)^{-\theta}\right]\right) \\
& \times\left((1+\lambda)-\lambda\left[1-(1+\gamma x)^{-\theta}\right]\right)^{(j-1)} \\
& \times\left\{\left(1-\left[1-(1+\gamma x)^{-\theta}\right]\right.\right. \\
& \left.\left((1+\lambda)-\lambda\left[1-(1+\gamma x)^{-\theta}\right]^{\alpha}\right)\right\}^{n-j}
\end{aligned}
$$

Therefore, the pdf of the largest order statistic $X_{(n)}$ is given by

$$
\begin{aligned}
& f_{X_{(n)}}(x) \\
& =n \frac{\theta \gamma\left[1-(1+\gamma \mathrm{x})^{-\theta}\right]^{\mathrm{n}-1}}{(1+\gamma \mathrm{x})^{(\theta+1)}} \\
& \times\left(1+\lambda-2 \lambda\left[1-(1+\gamma x)^{-\theta}\right]\right) \\
& \times\left((1+\lambda)-\lambda\left[1-(1+\gamma x)^{-\theta}\right]\right)^{(n-1)}
\end{aligned}
$$

and the pdf of the smallest order statistic $X_{(1)}$ is given by

$$
\begin{aligned}
& f_{X_{(1)}}(x) \\
= & n \frac{\theta \gamma\left(1+\lambda-2 \lambda\left[1-(1+\gamma x)^{-\theta}\right]\right)}{(1+\gamma \mathrm{x})^{(\theta+1)}} \\
\times & \left\{1-\left[1-(1+\gamma x)^{-\theta}\right]\left(\begin{array}{c}
(1+\lambda) \\
-\lambda\left[1-(1+\gamma x)^{-\theta}\right]
\end{array}\right)\right\}^{n-1}
\end{aligned}
$$

Note that $\lambda=0$ yields the order statistics of the Lomax distribution.

\section{Concluding Remarks}

In the present study, we have introduced a new generalization of Lomax distribution called the transmuted Lomax distribution. The subject distribution is generated by using the quadratic rank transmutation map and taking the Lomax distribution as the base distribution. Some mathematical properties along with estimation issues are addressed. The hazard rate function and reliability behavior of the transmuted Lomax distribution shows that 
the subject distribution can be used to model reliability data. We expect that this study will serve as a reference and help to advance future research in the subject area.

\section{References}

[1] Aryal, R. G. and Tsokos, P. C. On The Transmuted Extreme Value Distribution With Application. Nonlinear Analysis: Theory, Methods and Applications, 71, 1401-1407, 2009.

[2] Aryal, R. G. and Tsokos, P. C. Transmuted Weibull Distribution: A Generalization of the Weibull Probability Distribution. European Journal of Pure and Applied Mathematics, 4(2), 89-102, 2011.
[3] Aryal, R. G. Transmuted Log-Logistic Distribution. Journal of Statistics Applications \& Probability. No. 1, 11-20, 2013.

[4] Brent, M. Engineering With Mathcad. Butterworth-Heinemann. Oxford, 2006.

[5] Kenney, F. J. and Keeping. S. E.). Mathematics of Statistics. Princeton, NJ, 1962.

[6] Lomax, K.S. Business failures: Another Example of The Analysis of Failure data. Journal of the American Statistical Association 49, 847-852, 1954.

[7] Moors, A. J. A Quantile Alternative For Kurtosis. Journal of the Royal Statistical Society, D, 37, 25-32, 1998.

[8] Shaw, W and Buckley, I. The Alchemy of Probability Distributions: Beyond Gram- Charlier Expansions, and a SkewKurtotic-Normal Distribution From a Rank Transmutation Map. Research report, 2007. 